\title{
ANÁLISE DA IMPLANTAÇ̃̃O DO SISTEMA FECHADO SIMPLIFICADO DURANTE A ASSISTÊNCIA DE ENFERMAGEM AOS CLIENTES EM DIÁLISE PERITONEAL INTERMITENTE MANUAL *
}

\author{
Rosângela Aguilar Carrilho Guimarães **
}

\begin{abstract}
RESUMO - A autora relata a experiência realizada numa unidade de depuração extra-corpórea de um hospital militar, na cidade do Rio de Janeiro com a criação do Sistema Fechado Simplificado em diálise peritoneal intermitente (DPI) manual, a partir do antigo e convencional equipo de DPI manual. O novo sistema fechado simplificado 'foi testado pela enfermeira, em um grupo de 17 clientes no perlodo de 1986 a 1989. O antigo equipo convencional foi usado em 11 clientes no perlodo de 1982 a 1985. São apresentados e comentados os beneficios oriundos do novo sistema em comparação ao sistema antigo.
\end{abstract}

\begin{abstract}
The authoress reports the experience realized in a out-body depuration unif of a military hospital, in Rio de Janeiro, with the creation of the Simplified Closed System in manual internittent peritoneal dialysis, from the old and convetional manual DPI device. The new simplified closed system was tested by nurse on a group of 17 patients from 1986 to 1989. The old conventional devide was used on 11 patients from 1982 to 1985. The beneficesproceeding the new system are reported and discussed by comparing to the old device.
\end{abstract}

\section{INTRODUÇĀO}

A dialise peritoneal intermitente (DPI) é um método de depuração extra-corpórea, bastante utilizado pela nefrologia, devido à sua grande facilidade de execução e menor custo que outros métodos de depuração, apesar de sua duração ser mais longa e de efeito mais tardio.

A DPI manual é um método de substituição da funçấo renal que primeiro se destaca entre os outros, como bem assinalam Druker, Parsons e Maher citados por NOLPH': "A dialise peritoneal tem se expandido amplamente tanto em hospitais grandes, como nos pequenos, no tratamento da insuficiência renal grave". Ainda, segundo este autor, "apresenta a vantagem de que, normalmente, $e$ fácil iniciá-la e de um modo rápido".

No que concerne às complicações da DPI manual, podemos citar várias, porém é mais séria e comum delas, é a peritonite.

Para LEEHEY, GANDHI \& DAUGIRDAS ${ }^{2}$, a peritonite é o tendão de Aquiles da diálise peritoneal, apesar de sua incidência estar se reduzindo.

Segundo ALFRED et aliii $^{3}$, a frequêencia com que surge a peritonite varia com o grau de observação das técnicas assépticas e do tipo de dialise peritoneal que for executada. Por isso, a iniciativa de criar o sistema fechado simplificado em DPI manual surgiu da necessidade de controlar e diminuir a inciência de peritonite, além, de melhor dinamizar a assistência de enfermagem.

A simplificação do material de dialise também se faz cada vez mais premente, como bem afirmam LAZARU \& KJELLSTRAND": "O estudo da cinética da diáiise peritoneal proveu justificativa cientiffica para este procedimento e sua popularidade cresceu com a viabilidade de sistemas fechados que utilizem líquidos de diálise e catéteres estéreis para punções peritoneais repetidas".

Ainda, segundo MOYSÉS NETO ${ }^{5}$, a utilização da diálise peritoneal para clientes crônicos tem aumentado devido aos grandes avanços nos últimos anos, principalmente, o desenvolvimento do catéter de Tenckhoff e a prótese de Deanne, além do aparecimento dos sistemas fechados e automáticos.

KHANA, .OREOPOULOS ${ }^{6}$ citam os catéteres peritoneais permanentes e o temporário, descrevendo a técnica de implantação dos mesmos na cavidade abdominal.

Atentando para os fatores que interferem na execução da diálise peritoneal, foi desenvolvido um sistema simplificado para DPI manual e partir do antigo equipo, sendo tal sistema usado em um grupo de clientes.

Ocorreu menor percentual de peritonite no grupo de clientes, os quais usaram o novo sistema fechado simplificado em relação ao grupo que usava o equipo antigo. Além disso, tornou-se claro o surgimento de benefícios para o cliente e para a equipe de enfermagem.

\section{MATERIAL E MÉTODOS}

O experimento foi feito a partir do aperfeiçoamento do antigo equipo convencional de DPI manual (Figura 1).

\footnotetext{
* Premio Zaira Cintra Vidal - 1\% lu gar - 42\% Congresso Brasileiro de Enfermagem - Natal-RN.

** 1o Tenente-Enfermeira da Unidade de depuração extra-corpórea do Hospital da Força Aérea do Galeão. Enfermeira da Divisảo Médica da Câmara Municipal do Rio de Janeiro.
} 


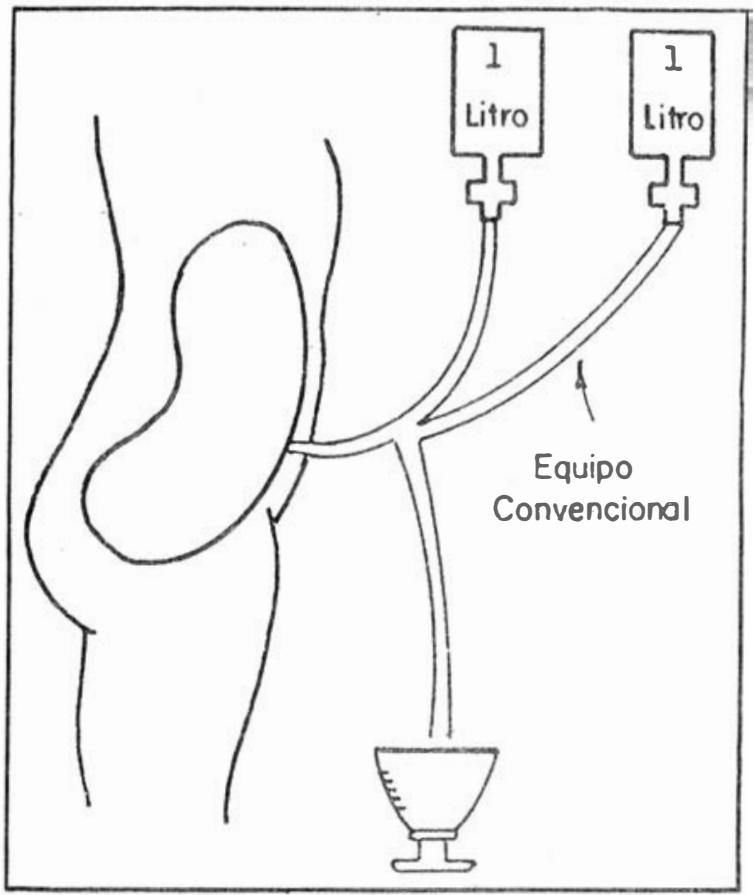

Figura 1

A realização do projeto foi gradual e fruto de observação, dividido em 3 fases.

\section{Fase: Montagem do Sistema Fechado}

Foi iniciado a criação do sistema fechado simplificado utilizando materiais disponíveis no setor de diálise, e de fácil aquisição, como:

- 01 equipo convencional de DPI manual;
- 01 sistema fechado de urina;

- 01 pedaço de borracha estéril (retirada de um equipo de soro estéril para servir de conecção;

-01 par de luvas esterelizadas;

- 02 frascos de 1 litro de solução de diølise peritoneal.

Com estes materiais, foi montado o sistema fechado simplificado, através da conecção do sistema fechado de urina ao antigo equipo (Figura 2).

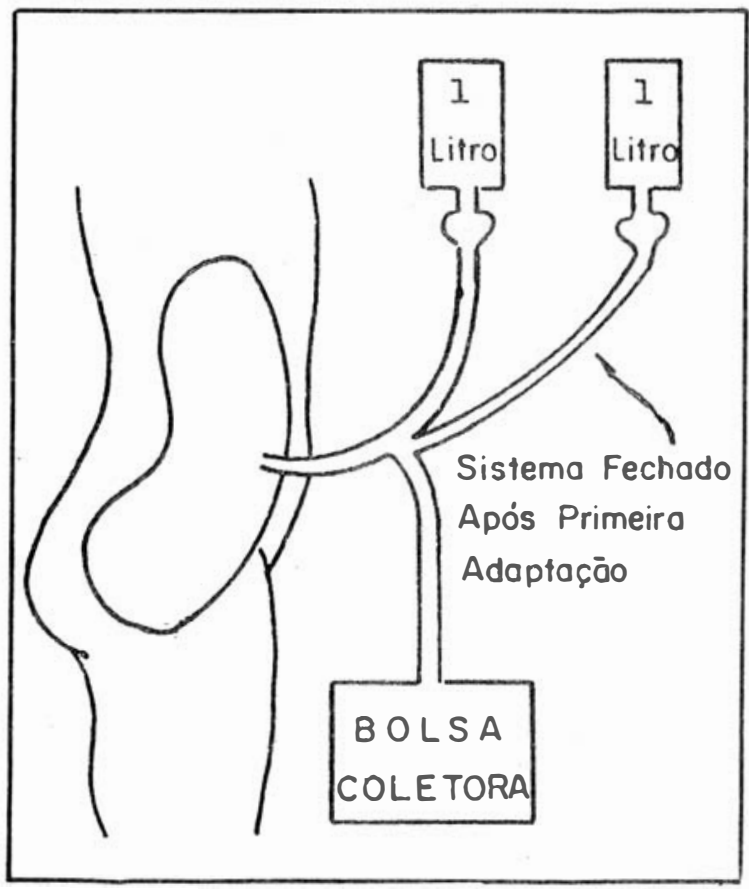

Figura 2 
Foi utilizado para isso, técnicas assépticas, incluindo o uso das luvas esterelizadas.

\section{2a Fase: Simplificação do Sistema Fechado}

Através da continuada vigilância e utilização do sistema fechado, foi percebido que o mesmo podia ser melhor aperfeiçoada, englobando os dois litros da solução de diálise em um só frasco (Figura 3).

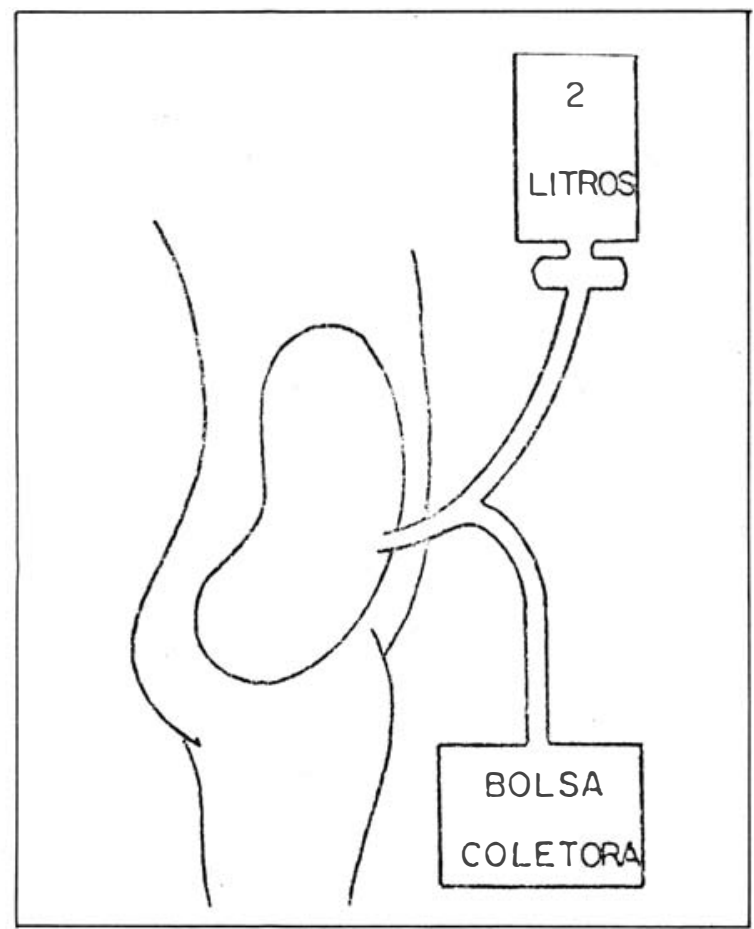

Foi contactada uma fábrica de produtos dialíticos e a mesma se prontificou a produzir o protótipo do sistema fechado simplificado de DPI manual.

\section{Fase: Implementação do Sistema Fechado Simplificado}

A fabricação em série do sistema fechado simplificado, após vários testes com o protótipo, possibilitou a definitiva utilização do sistema.

\section{RESULTADOS E DISCUSSÃO}

Para comprovar a efetividade e superioridade do sistema fechado simplificado, em relação ao equipo convencional, foi feito um levantamento dos pacientes que se submeteram a DPI manual.

O período de utilização do equipo convencional em DPI manual foi de 1982 a 1985 . O sistema fechado simplificado foi testado e utilizado no período de 1986 a 1989.

A clientela atendida foi composta de militares e seus dependentes que sofreram de insuficiência renal, necessitando de DPI manual. Isso explica o pequeno universo pesquisado (28 pacientes), quando comparado com a população civil.
A média de sessões de DPI manual por paciente foi a mesma nos dois períodos pesquisados (Diagrama 1), concluindo-se que a probabilidade do surgimento de peritonite foi igual para os dois grupos.

Em relação à peritonite, foi verificado que seu aparecimento foi maior com o equipo convencional, do que com o sistema fechado simplificado (Diagrama 2 e 3).

O percentual de peritonite com o equipo convencional foi bem superior ao ocorrido com o sistema fechado simplificado (Diagrama 4).

Com a redução do índice de peritonite, houve menor custo financeiro para o paciente e para a instituição.

O sistema fechado simplificado pode ser facilmente adaptado aos catéteres em uso na atualidade. Ocorreu maior incidência de peritonite nos pacientes que utilizaram o catéter rígido (Diagrama 5). Isto talvez se deva às múltiplas punções abdominais, necessárias à instalação do mesmo. A frequente manipulação da cavidade abdominal traz maior risco da contaminação.

A simplificação do equipo de DPI facilitou mais a sua montagem. Deve-se também, à simplificação do equipo, uma economia nos movimentos para execução da DPI, reduzindo assim o seu tempo de duração.

A existência da bolsa coletora propiciou uma melhor observação do fluxo e do aspecto do líquido drenado. Alé, disso, a equipe de enfermagem obteve maior liberdade e segurança, pois não ha' mais o risco do líquido drenado ser desprezado no chão. Também, possibilitou a colheita asséptica de amostra do líquido drenado.

\section{CONCLUSÃO}

pela observação e estudo realizado, ficou constado que houve uma melhora na otimização do padrão de assistência de enfermagem prestada aos clientes na unidade de depuração extra-corporea.

No campo aḍinistrativo, observamos redução no custo do tratamento:

1 - pela minimização da incidência de peritonite, com suas implicações referentes ao bem-estar do cliente.

2 - diminuição do tempo de permanência do cliente no hospital, e em consequência a diminuição do custo leito-dia.

Nas condições de trabalho da equipe multiprofissional, evidenciou-se benefícios, já que o paciente com infecção traz várias implicações nos diversos âmbitos assistenciais.

No campo técnico-profissional, evidenciou-se:

1 - simplificação do equipo convencional já existente;

2 - melhor observação do fluxo e aspecto do líquido de drenagem;

3 - fácil adaptação do sistema fechado aos catéteres, comumente usados;

4 - Colheita asséptica de amostra do líquido drenado para exame laboratorial. 
DIAGRAMA 1

Sessóes de DPI Manual

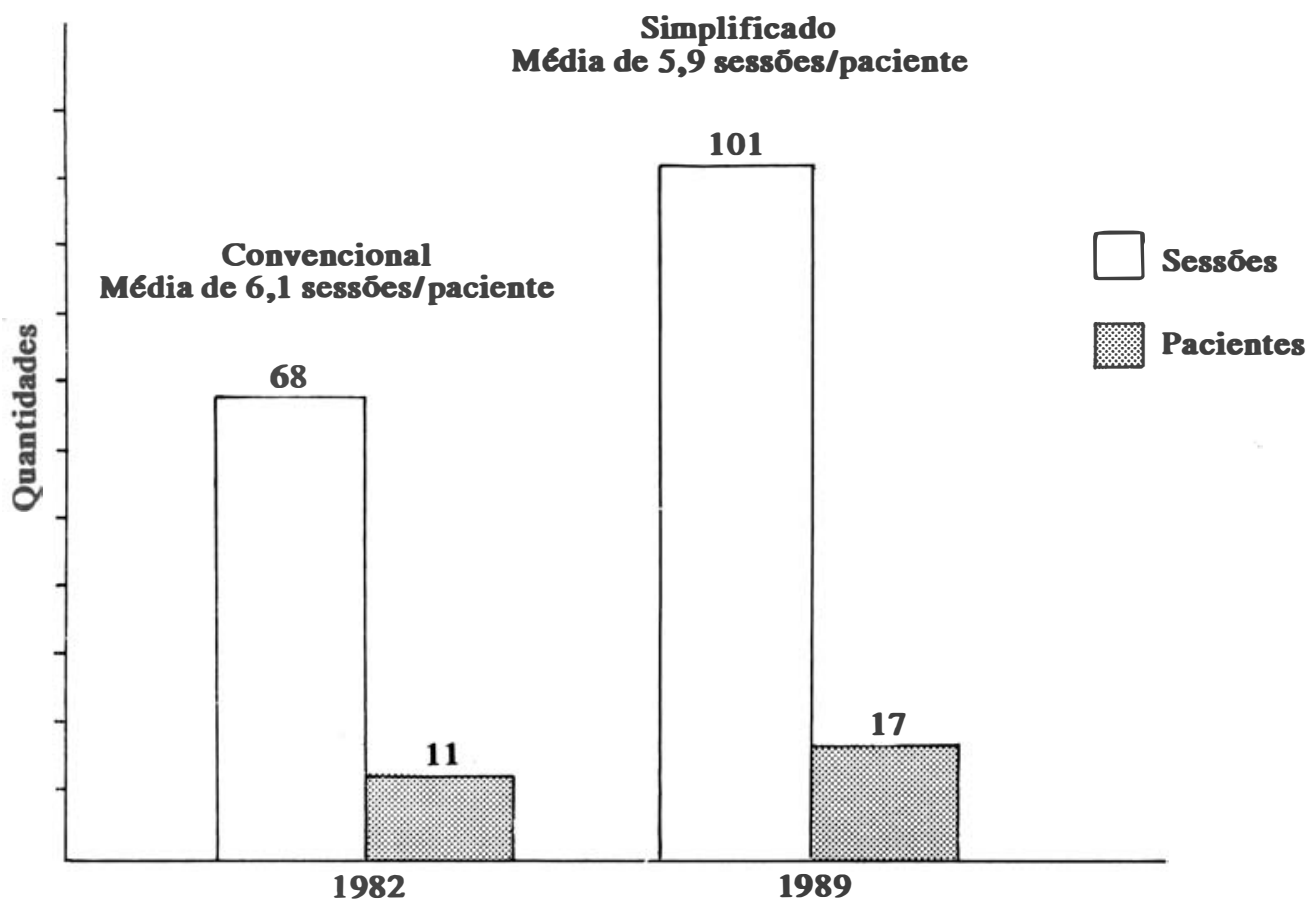

DIAGRAMA 2

Ocorrencia de Peritonite

Sistema Fechado Simplificado

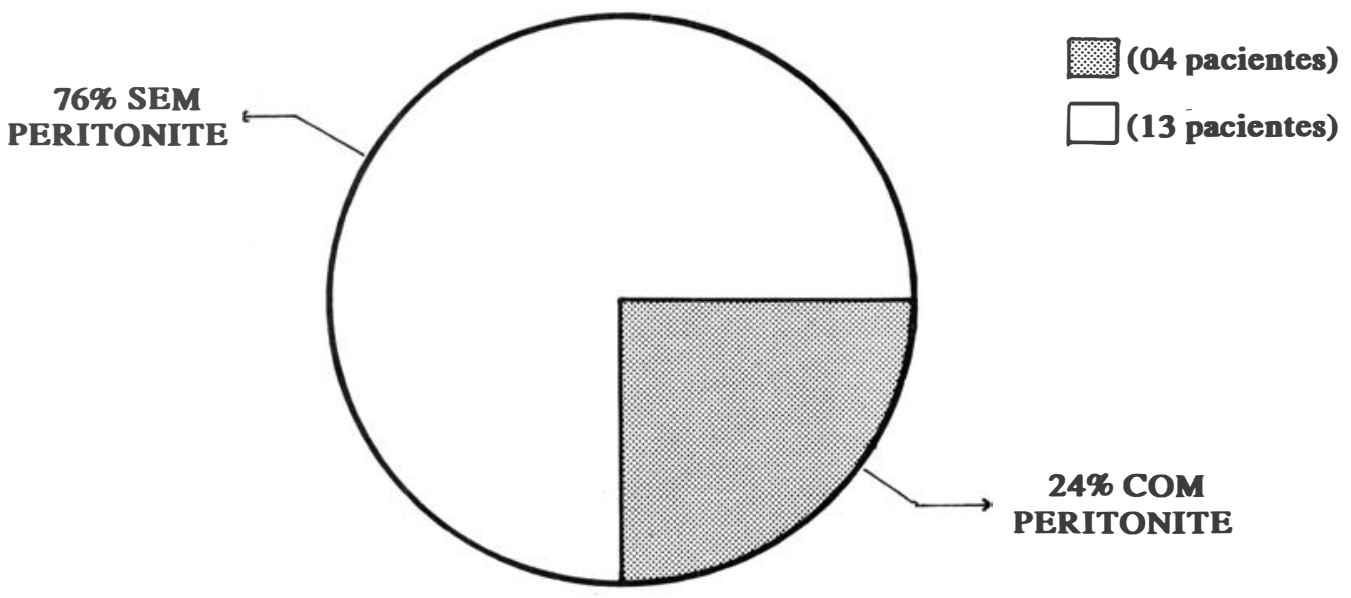

OBS: 1 caso entre os 4 com peritonite, já iniciou o tratamento com-infecção instalada, devido a programa anterior de CAPD. 


\section{DIAGRAMA 3}

\section{Ocorrencia de Peritonite}

Equipo Convencional

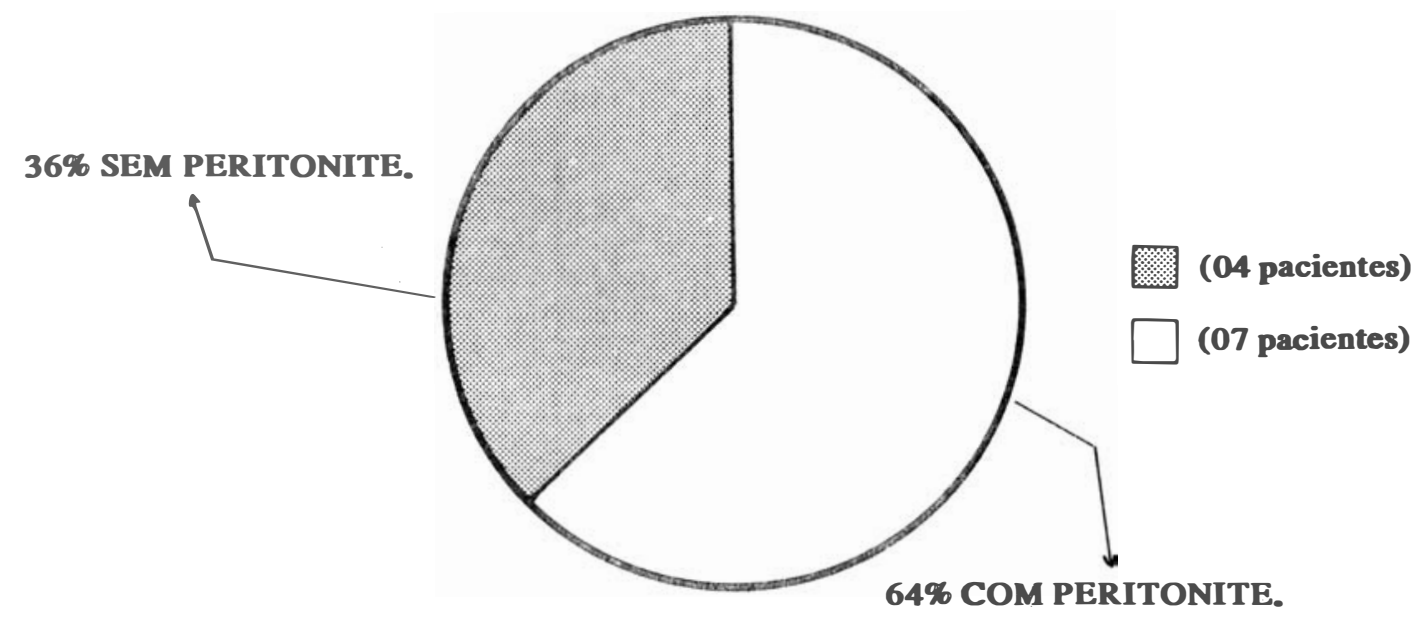

OBS: 2 casos entre os 7 com peritonite, já iniciaram o tratamento com infecção instalada, devido a programa anterior de CAPD.

\section{DIAGRAMA 4}

Comparaçāo da Ocorréncia de Peritonite

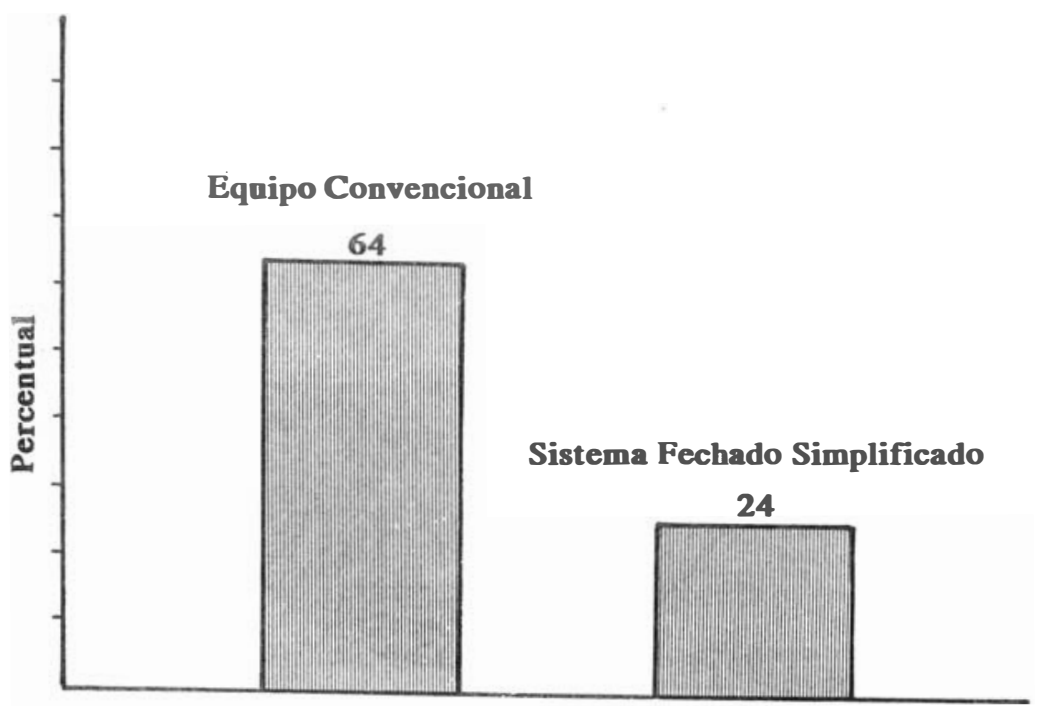




\section{DIÄGRAMA 5}

\section{Catêteres metilizados com o Sistema Fechado Simplificado}

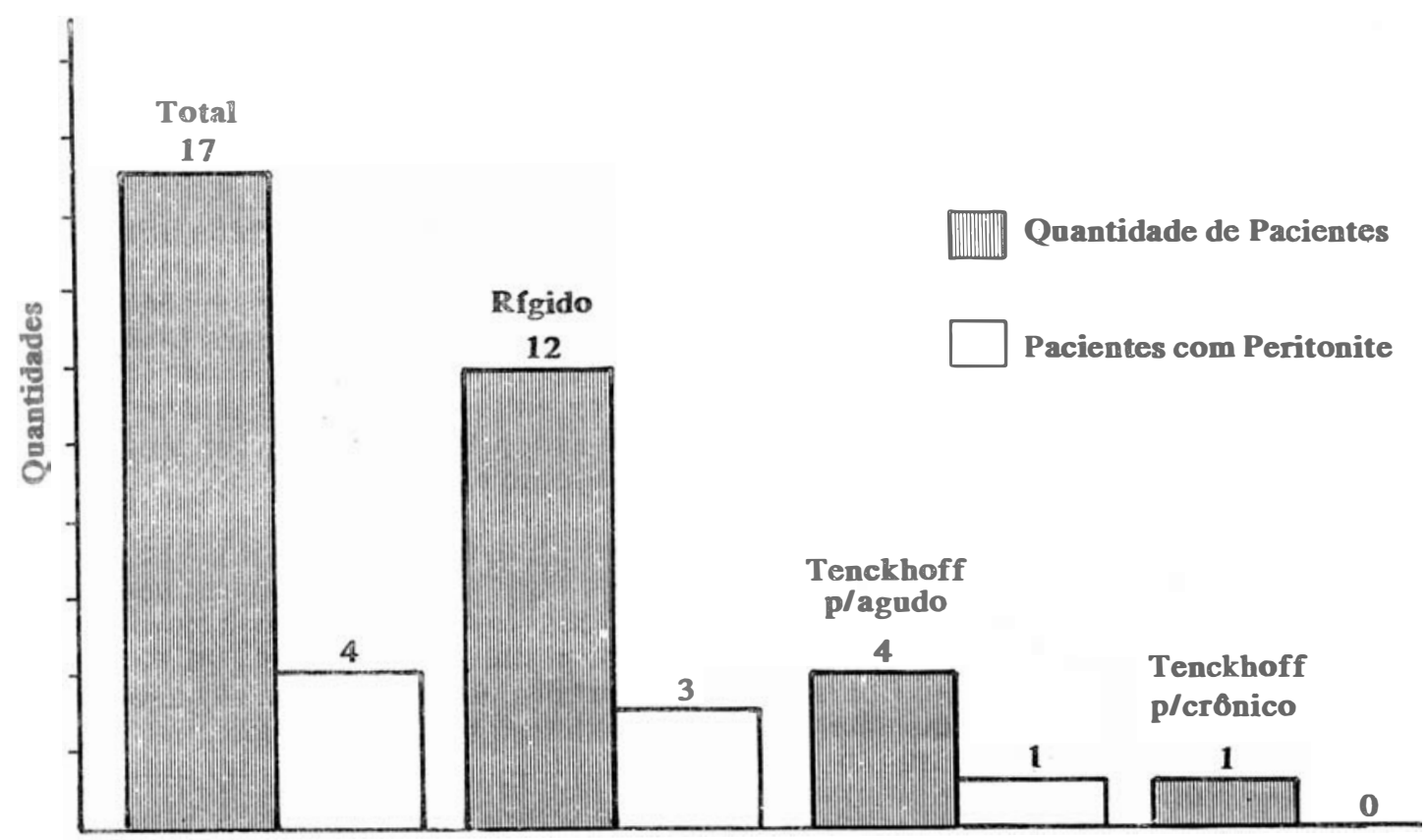

\section{REFERÊNCIAS BIBLIOGRÁFICAS}

1. NOLFH, K.D. Diálise Peritoneal. In: DRUKKER, W.PARSONS, F.M. MAHER, J.F. Sustitucion de la funcion renal por dialisis, Barcelona: Editorial, 1982. p. 253-85.

2. LEEHEY, D.J., GANDHI, V.C. DAUGIRDAS, J.T. Peritonitis. In: DAUGIRDAS, J.T.\& ING T.S. Handbook of Dialuysis. 1 ed. Boston/Toronto: Little Brow and company, 1988. p.252-71.

3. ALFRED, $\mathrm{H}_{a}$. et alii. Treatment of renal failure. In: ROSE, B.D. Pathophysiology of renal disease. Boston: Mc Graw-Hill Inc. 1981. p. 507-13.
4. LAZARUS, J.M. \& KJELLS TRAND, C.M. Dialysis: medical aspects. In: BRENNER, B.M. \& RECTOR, F.C. The Kidney, 2 ed. Philadelphia: W.B.Saunders C., $1981, v .2 . p .2490-525$.

5. MÓYSES NETO, M. Diálise Peritoneal. In. FERRAZ, A.S. et alii. Manual de tratomento da insuficiência renal aguda e crônica terminal. Rio de Janeiro: Ed. Interamericana, 1981, p.95-115.

6. KHANA, R. OREOPOULOS, D.G. Diálise Peritoneal. In: LEVINE, D.Z. Nefrologia - cuidados do paciente com doenças renais. São Paulo: Roca, 1985. p. 388-93. 